\title{
ACRODERMATITIS ENTEROPATHICA: A CASE REPORT
}

\author{
Nabin Bhakta Shakya ${ }^{1}$, Sudarsan Lal Rajbhandari ${ }^{1}$, Sagar Mani Jha ${ }^{1}$ \\ ${ }^{1}$ Department of Dermatology and Venereology Shree Birendra Hospital
}

\begin{abstract}
Acrodermatitis enteropathica is rare congenital autosomal recessive disorder. Premature are particularly risk and disease is caused by zinc deficiency due to a specific absorptive defect. This disease manifests in child as peevish, withdrawn and photophobia and develop vesicobullous dermatitis on hands, feet and perioroficial areas and alopecia. Diarrhea is often present. This also presents as shunted growth and decrease resistance to infection. Poor wound healing.A low plasma zinc level and Serum alkaline phospahtase are useful indicator of zinc status.
\end{abstract}

Key words: acrodermatitis enteropathica, vesicobullous dermatitis, zinc deficiency.

\section{INTRODUCTION}

Acrodermatitis enteropathica is the rare autosomal recessive disorder with prevalence of 1:500000. Premature are particularly at risk as this disease is caused by zinc deficiency due to a specific absorptive defect. Although the cause of absorptive defect is unknown, the genetic defect has been mapped to $8 q 24$ and the defective gene identified as SLC39A4, which encodes the zinc transporter Zip4. The disease typically starts after weaning or earlier if the infant is not given breast milk. However, clinical zinc deficiency may occur in full term and premature infants still breastfeeding. Usually, Acrodermatitis Enteropathica (AE) presents soon after fourth to tenth week of life in infants who are not breast fed. The classic featrues of AE include alopecia, diarrhea, lethargy and an acute eczematous and erosive dermatitis favoring acral areas- perioral, periocula, anogenital, hands and feet. The cutaneous findings are highly characteristic and often present initially as a non specific acrally distributed, symmetric, eczematous dermatitis, over time, bullae and erosions with a characteristic peripheral crusted border develop. Patients also appear to be predisposed to systemic infections as a result of impaired cell mediated. Delayed wound healing, acute paronychia, conjunctiviti, bleepharitis and photophobia may also be observed. Diarrhea may be prominent but is not seen in all cases. If untreated, the disease is fatal. A low plasma zinc level is the gold standard for diagnosing zinc deficiency. Serum alkaline phospahtase, a zinc dependent enzyme is another useful indicator of zinc status, as AP may be low even when plasma zinc levels are low normal, serum AP will increase with zinc supplementation, thus confirming the diagnosis. Patents with $\mathrm{AE}$ require lifelong treatment with zinc ${ }^{1,2,3}$.

\section{CASE REPORT}

A 7 years boy presented with erythematous scaly lesion in perioral, fingers, hands and perianal region. The lesions started since the child was 11 months and gradually increased in size, and the new lesion was noted on knee, foot and around anal area, in course of time there was involvement around mouth. Similarly there was gradual loss of hair in the scalp with photophobia. The lesion was occasionally pruritic. Patient underwent treatment

\footnotetext{
Correspondence :

Dr. Nabin Bhakta Shakya, Department of Dermatology \& Venereology, Shree Birendra Hospital, Nepal.

Email: docnabin@yahoo.com

Cell No.: 9841169123
} 
at various centers and various drugs were used, mostly moderate to potent topical steroid and salicylic acid. Although the vitamin syrup containing iron and zinc gave the best treatment response, this treatment was not continued for a long time. Although no abnormal perinatal history was noted, parents noted the growth was slow in comparision to other children of similar age group. On examination patient is irritable and restless. On skin examination there was erythematous scaly plaque with ill defined margin at hand, face, abdomen, periocular and perianal areas, multiple erosion and ulcer with crusts noted at the plaque. Sparse fine hair at scalp. There were no nails changes, and other systemic examinations were normal. The laboratory investigation showed low plasma zinc and serum alkaline phosphatase level. Skin biopsy revealed hyperkeratosis, confluent parakeratosis, spongiosis and focal dyskeratosis. Patient showed dramatic improvement after intake of zinc $10 \mathrm{mg}$ bid.

\section{DISCUSSION}

Acrodermatitis enteropathica is a rare autosomal recessive disorder of zinc deficiency. Although a deficiency dermatitis caused by low dietary zinc has the exact clinical and histologic features of $A E$, the term $A E$ should be reserved only for genetic causes of zinc deficiency. In humans AE, first described by Danbolt and Closs in 19434, is the only known hereditary defect of zinc metabolism. Individuals with $\mathrm{AE}$ suffer from severe zinc deficiency derived from a defective uptake of zinc in the duodenum and jejunum ${ }^{5}$. AE usually presents in infancy within days if an infant is bottle-fed and soon after weaning in infants breast-fed, such as our patient. Several studies have tried to explain this well characterized difference ${ }^{6,7,8}$ and as of yet there is no consensus. Initially, the cause of $A E$ was unknown and the disease was often fatal. The link between zinc deficiency and human pathology was not realized until 30 years after the initial clinical description of $A E^{9,10,11}$. Homozygosity mapping of consanguineous Jordanian and Egyptian kindreds with $A E$ localized the genetic defect to $8 q 24^{12}$. Soon thereafter the defective gene was identified as SLC39A4 ${ }^{13,14}$, which encodes a ZIP protein, ZIP4.

Characteristic lesions of $A E$ and acquired deficiency dermatitis caused by low zinc include dry scaly eczematous plaques of the face, scalp and anogenital area, as well as generalized alopecia; none of which were present at
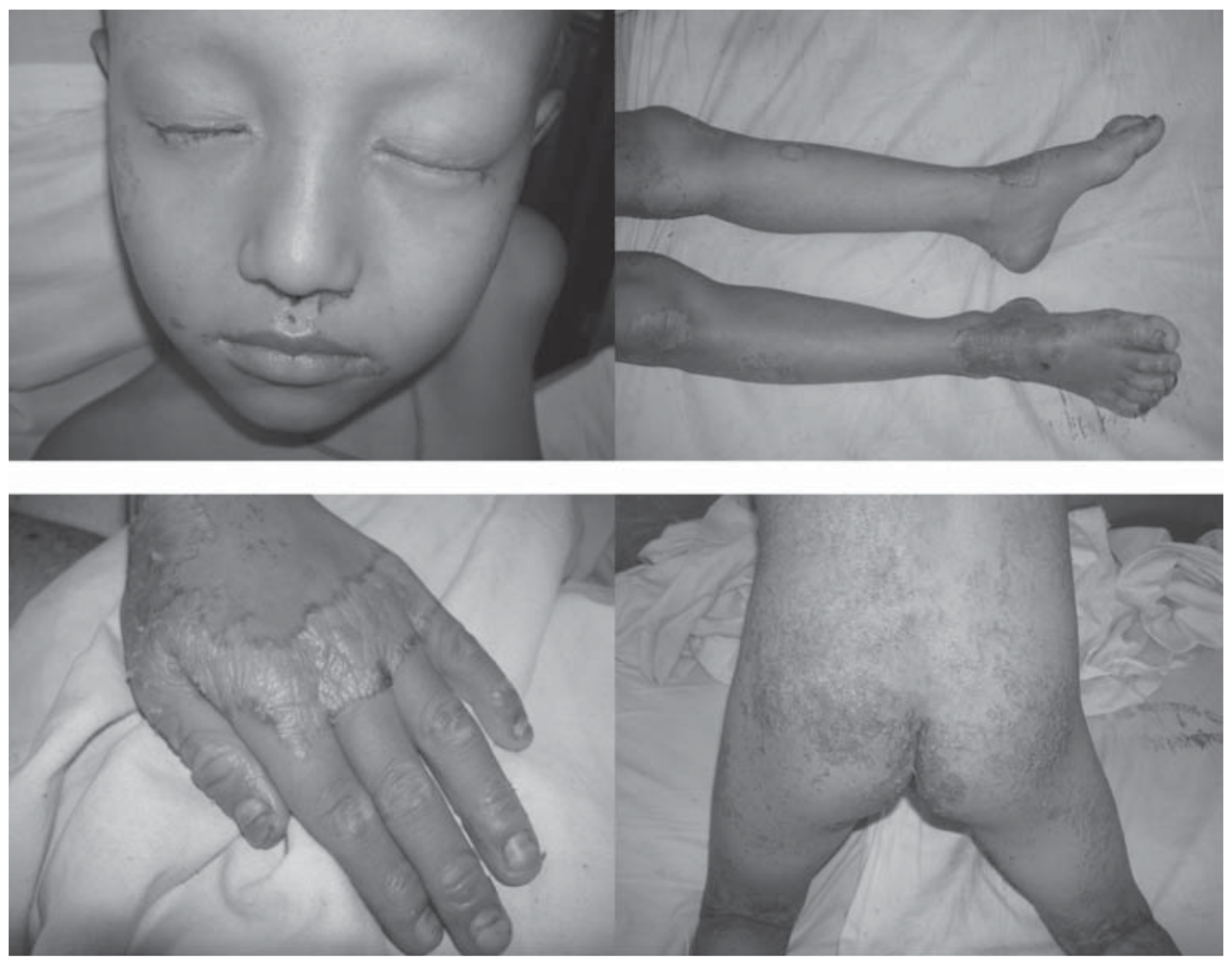

our patient's initial clinic visit. Establishing a diagnosis of zinc deficiency may be further complicated by the fact that, zinc levels may fall during states of inflammation. The mechanism for this has not been entirely elucidated but recently IL- 6 has been shown to upregulate the Zinc transporter Zip14.
Histopathologic and laboratory studies are helpful but cannot definitively make a diagnosis of zinc deficiency. For $\mathrm{AE}$, zinc replacement therapy should be started at 3 $\mathrm{mg} / \mathrm{kg} /$ day of elemental zinc (there is $50 \mathrm{mg}$ of elemental zinc per $220 \mathrm{mg}$ zinc sulfate). Serum zinc levels should be monitored and the dose of zinc sulfate should be adjusted appropriately. Patients may require a higher 
dose than $3 \mathrm{mg} / \mathrm{kg} /$ day of zinc sulfate to normalize their genetic defect of zinc metabolism. In deficiency dermatitis caused by low dietary zinc, replacement therapy should be initiated at $1 \mathrm{mg} / \mathrm{kg} /$ day of elemental zinc. Maverakis E, 2007 found in the department of dermatology of University of California at age 13, large well-defined and confluent erythematous plaques with grey to white scale over the extremities with lesions on periorally and perinasally. There was distal onycholysis of her toenails serum zinc level was $36 \mu \mathrm{g} / \mathrm{dl}$ (nl. 66-144 $\mathrm{gg} / \mathrm{dl}$ ). With zinc supplement her skin lesions almost entirely resolved within 1 month $^{15}$. Similarly, Shafi M, 1973 found fifteen cases of acrodermatitis enteropathica reported from S M H S Hospital Srinagar. There were 8 males and 7 females. The criterion of diagnosis of this condition was the clinical picture of symmetrical vesiculo-pustular dermatitis, in upper and lower limbs and periorificial regions. Thirteen cases had variable degrees of diarrhoea, 10 were having glossitis and stomatitis, while 4 had conjunctivitis, blepharitis and chronic paronychia. These children had low body weight, were apathetic and irritable ${ }^{16}$.

\section{CONCLUSION}

Acrodermatitis enteropathica is a rare autosomal recessive disorder of zinc deficiency. The genetic defect has been mapped to $8 q 24$ and the defective gene identified as SLC39A4, which encodes the zinc transporter Zip4. The diagnosis is made by way of clinical presentation together with histopathology and laboratory tests. Life long zinc therapy is required to reduce the symptoms and signs of disease.

\section{REFERENCES}

1. Irwin M., Braverman. Fitzpatrick's Dermatology in General medicine, 7th edition. 2008; 1215-1216.

2. Odom R. B., James W. B., Berger T. G. Andrews Disease of the skin, Clinical dermatology, 9th edition. 2000; 484.

3. D. J. Gawkrodger. Rook/Wilson/Ebbing- Text Book of Dermatology, 7th edition.2004; 57.103.
4. Danbolt, N., and K. Closs. 1943. Acrodermatitis enteropathica. Acta Derm Venereol 23:172.

5. Lombeck T, Schnippering HG, Ritzl F, Feinendegen LE, Bremer HJ. Letter: Absorption of zinc in acrodermatitis enteropathica. Lancet. 1975 Apr 12; 1(7911):855.

6. Eckhert CD, Sloan MV, Duncan JR, Hurley LS. Zinc binding: a difference between human and bovine milk. Science. 1977 Feb 25; 195(4280):789-90.

7. Hurley LS, Duncan JR, Sloan MV, Eckhert CD. Zinc-binding ligands in milk and intestine: a role in neonatal nutrition? Proc Natl Acad Sci U S A. 1977 Aug; 74(8):3547-9.

8. Cousins RJ, Smith KT. Zinc-binding properties of bovine and human milk in vitro: influence of changes in zinc content. Am J Clin Nutr. 1980 May; 33(5):1083-7.

9. Barnes PM, Moynahan EJ. Zinc deficiency in acrodermatitis enteropathica: multiple dietary intolerance treated with synthetic diet. Proc R Soc Med. 1973 Apr;66(4):327-9.

10. Moynahan EJ, Barnes PM. Zinc deficiency and a synthetic diet for lactose intolerance. Lancet. 1973 Mar 24; 1(7804):676-7.

11. Neldner $\mathrm{KH}$, Hambidge KM. Zinc therapy of acrodermatitis enteropathica. N Engl J Med. 1975 Apr 24; 292(17):879-82.

12. Wang K, Pugh EW, Griffen S, Doheny KF, Mostafa WZ, al-Aboosi MM, el-Shanti H, Gitschier J. Homozygosity mapping places the acrodermatitis enteropathica gene on chromosomal region 8q24.3. Am J Hum Genet. 2001 Apr; 68(4):1055-60. Epub 2001 Mar 12.

13. Kury S, Dreno B, Bezieau S, Giraudet S, Kharfi M, Kamoun $R$, Moisan JP. Identification of SLC39A4, a gene involved in acrodermatitis enteropathica. Nat Genet. 2002 Jul; 31(3):23940. Epub 2002 Jun 17.

14. Wang K, Zhou B, Kuo YM, Zemansky J, Gitschier J. A novel member of a zinc transporter family is defective in acrodermatitis enteropathica. Am J Hum Genet. 2002 Jul; 71(1):66-73.Epub2002May24.

15. Maverakis E, Lynch $\mathrm{P}$ and Fazel N. Acrodermatitis enteropathica. Dermatology Online Journal. 2007; 13 (3): 11.

16. Shafi $M$ and Ahmed SNS. Acrodermatitis enteropathica. IJDVL. 1973; 39 (1):33-34. 